\title{
A Transient Method Using Liquid Crystal for Film Cooling Over a Convex Surface
}

\author{
PING-HEI CHEN*, MIN-SHENG HUNG and PEI-PEI DING \\ Department of Mechanical Engineering, National Taiwan University, Taipei 10617, Taiwan
}

(Received 23 May 2000; In final form 27 May 2000)

\begin{abstract}
In order to explore the effect of blowing ratio on film cooling over a convex surface, the present study adopts the transient liquid crystal thermography for the film cooling measurement on a straight circular hole configuration. The test piece has a strength of curvature $(2 r / D)$ of 92.5 , pitch to diameter ratio $(P / D)$ of 3 and streamwise injection angle $(\gamma)$ of $35^{\circ}$. All measurements were conducted under the mainstream Reynolds number $\left(R e_{d}\right)$ of 1700 with turbulence intensity $(T u)$ of $3.8 \%$, and the density ratio between coolant and mainstream $\left(\rho_{c} / \rho_{m}\right)$ is 0.98 . In current study, the effect of blowing ratio $(M)$ on film cooling performance is investigated by varying the range of blowing ratio from 0.5 to 2.0. Two transient tests of different injection flow temperature were conducted to obtain both detailed heat transfer coefficient and film cooling effectiveness distributions of measured region. The present measured results show that both the spanwise averaged heat transfer coefficient and film cooling effectiveness increase with decreased blowing ratio.
\end{abstract}

Keywords: Convex surface; Film cooling; Liquid crystal thermography

\section{INTRODUCTION}

Film cooling is a technique of cooling gas turbine blades to protect them from high temperature gases. The technique could be used by injection of a film of cooling air onto the blade surface through discrete holes. These holes are typically inclined at approximately $30^{\circ}$ to $40^{\circ}$ with respect to the surface. Past film cooling studies have concentrated on film cooling effectiveness and heat transfer coefficient of flat surfaces. However, the flow through turbine passages will experience strong curvature effect, which is not observable in flow over flat surfaces.

Ito et al. (1978) conducted cooling film measurements on curved surfaces for a wide range of blowing rates by mass transfer analogy. They used a foreign gas injection technique to measure impermeable wall concentrations downstream of a row of injection holes and to obtain the impermeable-wall effectiveness. Their research showed that film cooling effectiveness of a convex

\footnotetext{
*Corresponding author. Tel.: 886-2-23621522 ext 11(0), Fax: 886-2-23644871, e-mail: phchen@ccms.ntu.edu.tw
} 
surface is higher than both flat and concave surfaces when the tangential component of injection flow momentum flux ratio $\left(I \cos ^{2} \gamma\right)$ is less than unity at injection angle between $0^{\circ}$ to $90^{\circ}$. Ko et al. (1986) measured the static pressure and the film cooling effectiveness of both convex and concave surfaces at different blowing ratios. Their experimental results suggested that the distance between cooling holes on the convex surface should be smaller than those on concave surface. A smaller blowing ratio should also be used on convex surface, because the recirculation is stronger on the convex surface than on concave surface even at low blowing ratio of 0.5 .

Schwarz et al. (1991) used a foreign gas injection technique to study the effects of strength of convex surface curvature on the film cooling performance. They considered three different strengths of curvature (ratio of radius of curvature to radius of injection hole), density ratios of 0.95 and 2.0, and blowing ratios of 0.3 to 2.7 . Their experimental results showed that cross-stream pressure gradient tends to shift film cooling jets onto the convex surface and away from the concave surface. At low blowing ratio, where both tangential and normal momenta are weak, the film cooling on a convex surface is more effective than a flat surface that has better performance than a concave surface. Goldstein et al. (1997) measured film cooling effectiveness on both convex and concave surfaces at different injection angles and blowing ratios by using mass transfer analogy. The flow field was visualized by an ammonia vapor injection system. They found that at very low blowing ratios, the injection flows were ejected gently into the boundary layer of mainstream, and the film cooling effectiveness would increase with blowing ratio independently of injection angle. As the blowing ratio is increased beyond the lift-off blowing ratio (the blowing ratio at which the cooling jets lift away from the surface and effectiveness begins to drop), the shallower injection angles perform better. The trajectory of shallower injection flows being somewhat closer to the surfaces than that of steeper injection flows.
Recently, some researchers have predicted the film cooling effectiveness by numerical method. Berhe et al. (1999a) used algebraic relations for the turbulent viscosity and the turbulent Prandtl number in a modified $k-\varepsilon$ turbulence model to study the effects of surface curvature on film cooling performance. Computations were performed at blowing ratios of $0.5,1.0$ and 1.5 with a density ratio of 2.0 along convex, concave and flat surfaces. Berhe et al. (1999b) also investigated the effect of several film cooling parameters including blowing ratio, injection angle, hole length, hole spacing and hole staggering. Lin et al. (1998) used a low-Reynolds number turbulence model to investigate film cooling of flat and convex surfaces. They showed that the injection flow lift-off phenomenon depends not just on the momentum flux ratio but also on the profile of boundary layer and the ratio of boundary layer thickness to hole diameter.

According to published studies, the environmental conditions must be well-controlled during measurements using the liquid crystal thermography. Camci et al. (1992) investigated the effects of the strength of the light source illuminating the heat transfer surface, the orientation of the light source with respect to the surface, the uniformity of coated liquid crystal layer, and the repeatability of the measured results. They suggested that the image capturing process should be performed with the same illumination angle and the illumination source should be fixed at a specific location for both the calibration process and measurement. Behle et al. (1996) showed that the calibration of camera and light source as the calibration step is very important to avoid an unreliable hue versus temperature relation. Besides the hue versus temperature relations that depend strongly on the coating thickness, the signal noise versus temperature relation was compared. They used both the TLC spray $(34 \mu \mathrm{m})$ and sheets to investigate the dependence of the hue values on variation of illumination and viewing angle for an off-axis and an on-axis camera and light source arrangement. 
Since the film cooling phenomena on curved surfaces are more complex than on flat surfaces, the detailed studies on local film cooling performance over the whole area of the curved surface are needed for the turbine blade design purpose. In this study, detailed heat transfer coefficient and film cooling effectiveness distributions are demonstrated over a convex surface by employing transient liquid crystal thermography (Vedula and Metzger, 1991; Ekked et al., 1997a, b and Chen et al., 1998). For the present experimental measurement, the streamwise injection angle $(\gamma)$ is $35^{\circ}$ and the spanwise injection angle is $0^{\circ}$. The blowing ratio $(M)$ was varied from 0.5 to 2.0 at a mainstream Reynolds number $\left(R e_{d}\right)$ of 1700 with turbulence intensity of $3.8 \%$, and the curvature strength $(2 r / D)$ of convex test piece is 92.5 .

\section{THEORY}

The present experiment used the thermochromic liquid crystal for the surface temperature measurement. Liquid crystal is very suitable to show the transient surface temperature, since their response is repeatable and their colors can be easily recorded with a video system. The local heat transfer coefficient over the liquid crystal coated surface without cooling film can be obtained by one-dimensional semi-infinite. The one-dimensional transient heat conduction equation, the convection boundary conditions, and the initial condition on the liquid crystal coated surface are:

$$
\begin{gathered}
k \frac{\partial^{2} T}{\partial z^{2}}=\rho_{s} C_{p_{s}} \frac{\partial T}{\partial t} \\
\text { at } z=0, \quad-k \frac{\partial T}{\partial z}=h\left(T_{w}-T_{f}\right), \\
\text { as } z \rightarrow \infty, \quad T=T_{i} \\
\text { at } t=0, \quad T=T_{i}
\end{gathered}
$$

where $k, C p_{s}$ and $\rho_{s}$ are respectively the thermal conductivity, the specific heat and the density of test piece. The analytic solution of Eq. (1) on the surface $(z=0)$ becomes:

$$
\frac{T_{w}-T_{i}}{T_{m}-T_{i}}=1-\exp \left(\frac{h^{2} \alpha t}{k^{2}}\right) \operatorname{erfc}\left(\frac{h \sqrt{\alpha t}}{k}\right)
$$

The film cooling over a surface involves three temperatures, including the mainstream temperature, coolant flow temperature and wall surface temperature. The mainstream temperature $\left(T_{m}\right)$ in Eq. (4) will be replaced by a film temperature $\left(T_{f}\right)$ during the data analysis. To find the unknown $T_{f}$ (in terms of known quantities $T_{m}$ and $T_{c}$ ), a nondimensional temperature is defined as the film cooling effectiveness:

$$
\eta=\frac{T_{f}-T_{m}}{T_{c}-T_{m}}
$$

Substituting Eq. (5) into Eq. (4) gives

$$
\begin{aligned}
T_{w}-T_{i}= & {\left[1-\exp \left(\frac{h^{2} \alpha t}{k^{2}}\right) \operatorname{erfc}\left(\frac{h \sqrt{\alpha t}}{k}\right)\right] } \\
& \times\left[\eta T_{c}+(1-\eta) T_{m}-T_{i}\right]
\end{aligned}
$$

The two unknown parameters are $h$ and $\eta$. In the present study, two film cooling tests with different $T_{c}$ have been completed. During measurements, the temperature rises of both mainstream and injection flow are functions of time, instead of step temperature change. Therefore, the Duhamel's Super-position should be used to modify the time varying temperature rises at inlets of both mainstream and injection flows.

Besides the heat transfer coefficient and film cooling effectiveness, heat flux ratio is another important parameter used to quantify the film cooling performance. The present study adopts the expression of heat flux ratio as defined by Ekkad et al. (1997b) given by

$$
\frac{q}{q_{o}}=\frac{h\left(T_{w}-T_{a w}\right)}{h_{o}\left(T_{w}-T_{m}\right)}=\frac{h}{h_{o}}\left(1-\frac{\eta}{\phi}\right)
$$

where $\phi$ is the overall cooling effectiveness. In current study, $\phi=0.6$ is used. 


\section{EXPERIMENTAL APPARATUS AND PROCEDURES}

The test section used for the experimental analysis of the film cooling effectiveness and heat transfer characteristics is shown in Figure 1. The experimental investigation was conducted in a wind tunnel with rectangular cross-section of $10 \mathrm{~cm} \times$ $5 \mathrm{~cm}$ and $\mathrm{a}$ bend of $135^{\circ}$. The wind tunnel consisted of a mix section, a uniform development section, and a curved test section as shown in Figure 1. The radii of curvature of the curved surfaces in the test section was $16.6 \mathrm{~cm}$. The mainstream velocity $\left(u_{m}\right)$ was kept at a constant value of $9.1 \mathrm{~m} / \mathrm{s}$. The velocity of mainstream was measured by a hot-film anemometer (Dantec, Flow Master 54N60). The free stream turbulence intensity was controlled by turbulence grid formed by cylindrical bars located upstream of the test section. The free stream turbulence was measured using a hot-wire anemometer, which was calibrated by TSI intelligent flow Analyzer (IFA-100) with constant temperature anemometer operation method.

The boundary layer was tripped at a distance of $10 \mathrm{~cm}$ upstream of the leading edge of injection

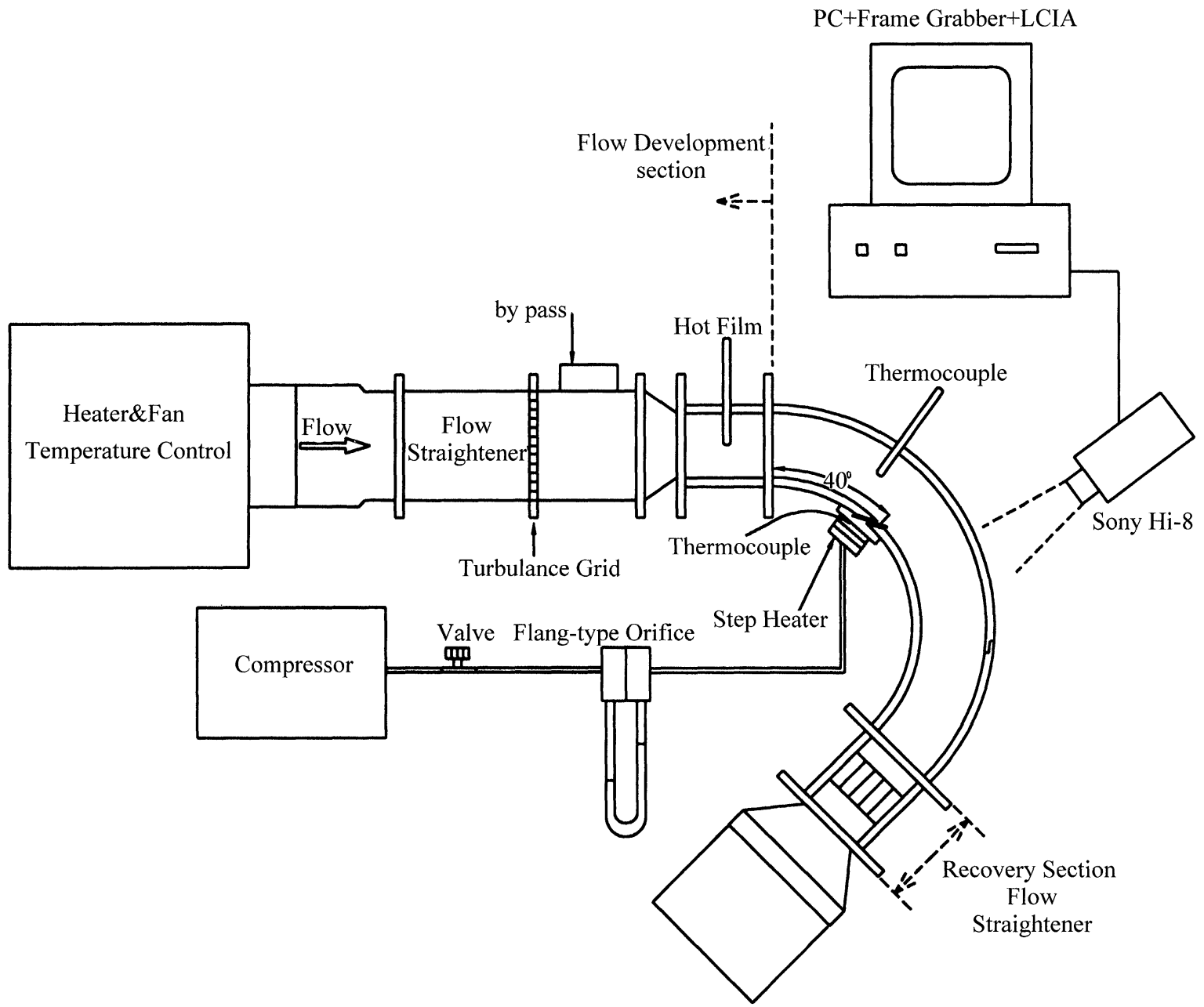

FIGURE 1 Schematic view of test facility. 
holes by a $1.5-\mathrm{mm}$-dia trip wire. The injection flow was injected through a single row of injection holes with hole spacing of $3 D$ along the spanwise direction. The hole diameter $(D)$ was $3.6 \mathrm{~mm}$ and the ratio of hole length to hole diameter $(L / D)$ was 3.5. The strength of curvature $(2 r / D)$ and the streamwise injection angle $(\gamma)$ were 92.5 and $35^{\circ}$ respectively, as shown in Figure 2. The plexiglas test surface was coated with black paint and a thin layer of thermochromic liquid crystal. The emissivity of the black paint is 0.94 . The injection flow was supplied by a reciprocating-type compressor that has a capacity of producing an air flow rate of $0.0018 \mathrm{~m}^{3} / \mathrm{s}$ at pressure of $7 \mathrm{~atm}$. A calibrated flange-type orifice was used to measure the mass flow rate of injection flow. The injection flow was heated by heating wires fixed at the inlet of the injection holes. The distance between heating wires and the injection holes is $205 \mathrm{~mm}$. The heating wire diameter is $1.2 \mathrm{~mm}$ and the ratio of the distance between the wires and the injection holes to the wire diameter is 170.8 . The mainstream temperature and the initial temperature on test surface were measured by T-type thermocouples, and a temperature recorder (Rustrak-Ranger II) records the time-varying temperatures during measurements.

The injection flow and mainstream were heated before the onset of measurement. When the temperature of injection flow and mainstream attained the desired experimental temperature, both injection flow and mainstream were diverted instantaneously into injection holes and test section. Meanwhile, the temperature recorder and $\mathrm{Hi}-8$ video recorded the temperature histories of both injection flow and mainstream, and the color-changing image of liquid crystal. Colorchanging images recorded on videotapes were then transmitted to a personal computer by a frame grabber and analyzed using image processing software. The transient time was taken through LCIA V3.0 (Liquid Crystal Image Analyzer Version 3.0).

The operating conditions are listed in Table I. The mainstream velocity measured by hot-film anemometer was $9.1 \mathrm{~m} / \mathrm{s}$ at $25 \mathrm{~cm}$ upstream of injection holes. The mainstream Reynolds number was 1700 based on the inlet diameter of injection
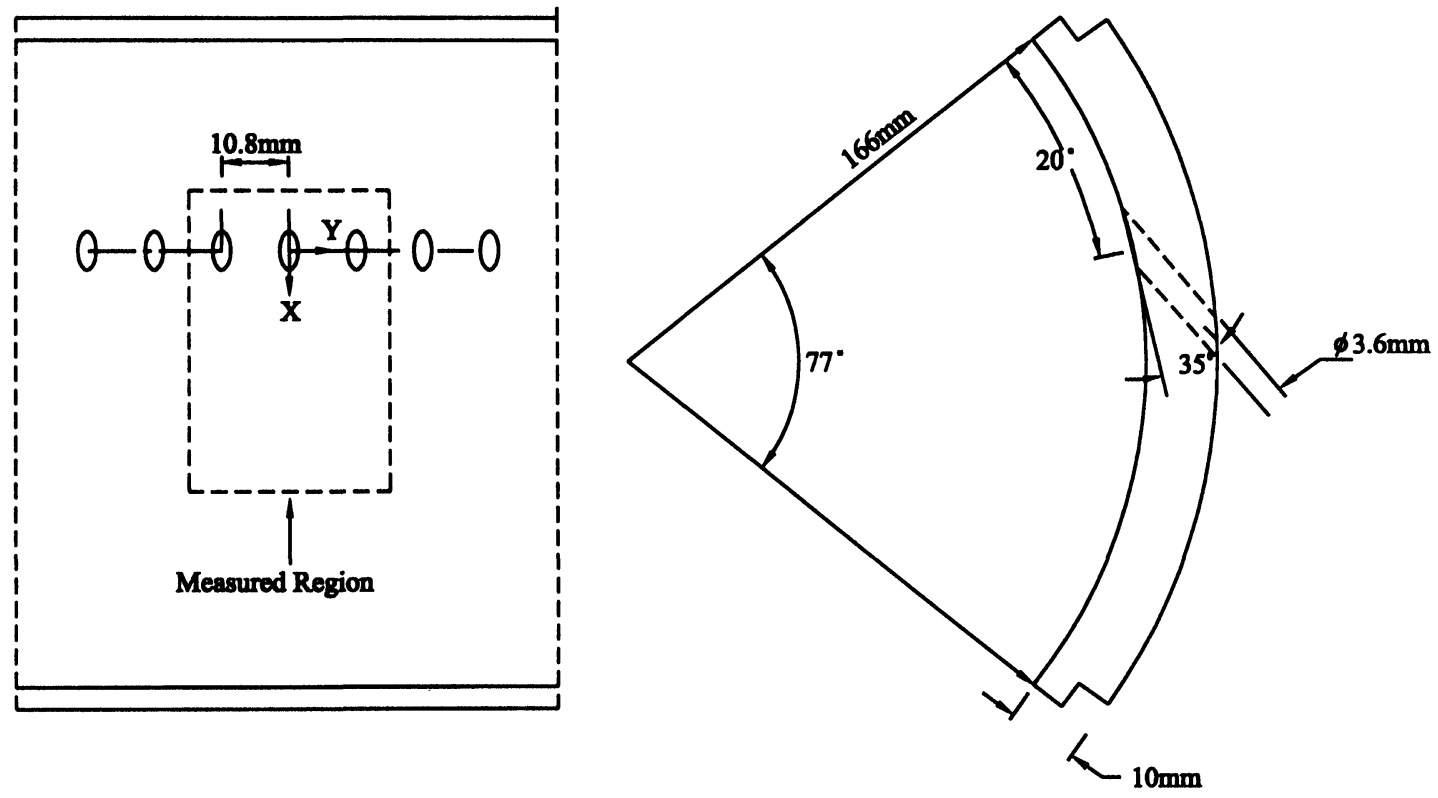

FIGURE 2 Convex test piece with simple hole configuration. 
TABLE I Test conditions for the measured results shown in Figure 3

\begin{tabular}{lccccccc}
\hline Authors & $P / D$ & $L / D$ & $\rho_{c} / \rho_{m}$ & $2 r / D$ & $\gamma$ & $\delta_{1} / D$ & $M$ \\
\hline Goldstein et al. $(1997)$ & 2.96 & 10 & 0.95 & 92.5 & $45^{\circ}$ & 0.5 & 1.0 \\
Schwarz et al. $(1991)$ & 3 & 10 & 0.95 & 94 & $35^{\circ}$ & 0.9 & 1.01 \\
The present study & 3 & 3.5 & 0.98 & 92.5 & $35^{\circ}$ & 0.12 & 1.0 \\
\hline
\end{tabular}

hole. The measured turbulence intensity was $3.8 \%$. Measurements were conducted at five different blowing ratios of $0.5,0.75,1.0,1.5$ and 2.0.

The estimated uncertainty in the effective data was \pm 5 percent estimated by the root mean square method by Moffat (1988). There are a few factors that affect the uncertainties of $h$ and $\eta$, including mainstream temperature, injection temperature, initial temperature, wall temperature, and the time of liquid crystal color change. For the experiments done in this study, the total uncertainties of $h$ and $\eta$ were respectively around $7.2 \%$ and $10.4 \%$ of their nominal values $\left(h=29.038 \mathrm{~W} / \mathrm{m}^{2} \mathrm{~K}\right.$ and $\eta=0.3232$ ). Furthermore, the estimated uncertainties for the Reynolds number, blowing ratio, mainstream turbulence intensity, and boundary layer displacement thickness were respectively $3.4 \%, 2.8 \%, 4.3 \%$ and $5.6 \%$.

\section{RESULTS AND DISCUSSION}

In Figure 3, the spanwise averaged film cooling effectiveness result is compared with the experimental results of Goldstein et al. (1997) and Schwarz et al. (1991). The present result is close to the result of Schwarz et al. The lower values in the result of Goldstein et al. might be due to the larger injection angle of $45^{\circ}$. Goldstein et al. stated that shallower injection angles have the better effectiveness near injection holes on a convex surface, as show in the comparison result of Figure 3.

Detailed distributions of local heat transfer coefficient ratio $\left(h / h_{o}\right)$ at four different blowing ratio of $0.5,1.0,1.5$ and 2.0 are shown in Figure 4 to demonstrate the influence of cooling film on a convex surface. At blowing ratio $(M)$ of 0.5 , it is obvious that $h / h_{o}$ increases just downstream of

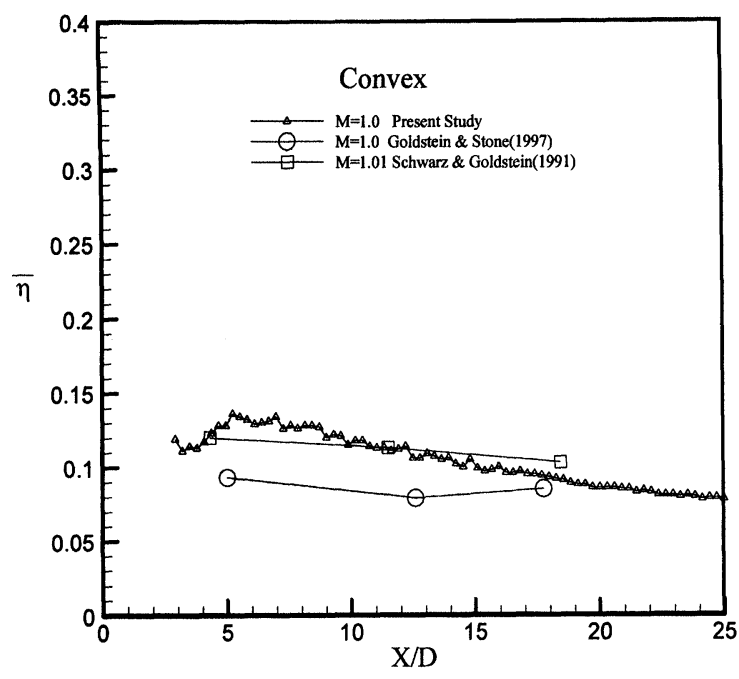

FIGURE 3 Comparison with prior measured results of spanwise averaged film cooling effectiveness over convex surface with simple hole configuration.

(a)

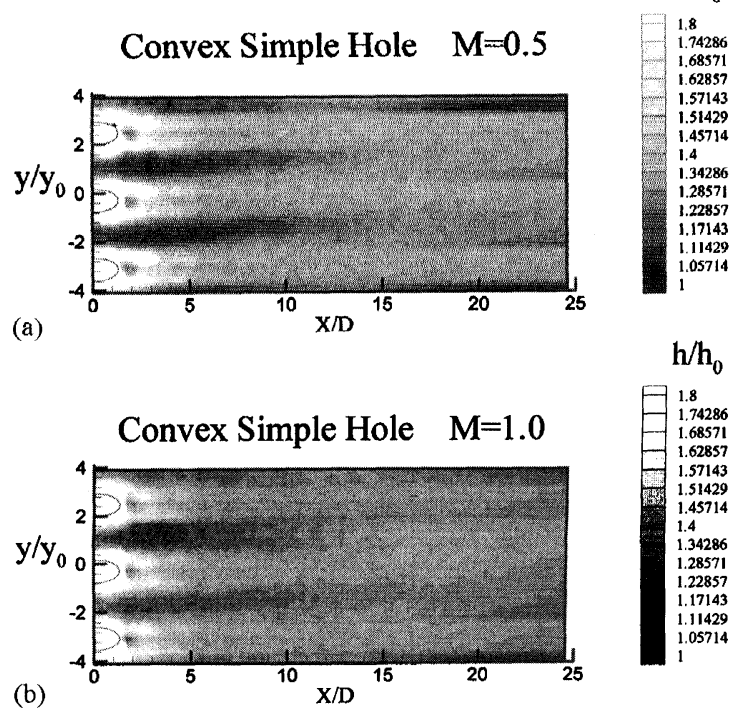

FIGURE 4 Local heat transfer coefficient ratio distributions at (a) $M=0.5$; (b) $M=1.0$; (c) $M=1.5$; (d) $M=2.0$. 

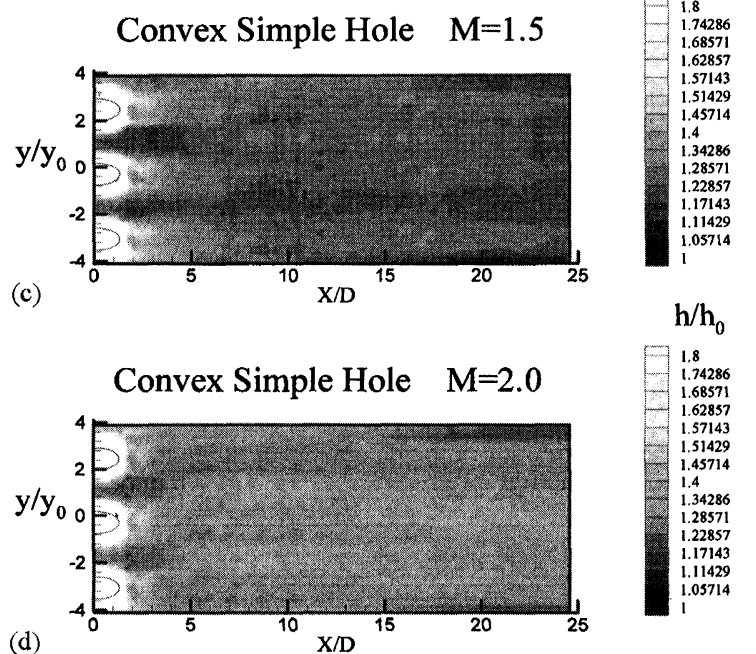

FIGURE 4 (Continued).

injection holes. Moreover, high heat transfer regions around both sides of injection holes, which may be caused by counter-rotating vortex pairs at the exit of holes is clear too. Therefore, the $h / h_{o}$ on the centerline regions downstream $(3<X / D<8)$ of injection holes becomes lower than the regions adjacent to it because the counter-rotating vortex pairs has brought the hot gas away from the surface.

The centerline region with $h / h_{o}>1.8$ downstream of injection hole for $M=1.0$ is smaller than at $M=0.5$, as shown in Figure 4(b). Because of higher $h / h_{o}$ at a low blowing ratio of 0.5 , it is expected that the spanwise averaged value of $h / h_{o}$ at $M=1.0$ will be lower than the value at $M=0.5$. In Figure 4(c), when blowing ratio increases to 1.5, the increased mass of injection flow will increase the mainstream boundary layer thickness and decrease $h / h_{o}$ at downstream regions of $X / D>7$. When the blowing ratio is increased to 2.0 as shown in Figure 4(d), the $h / h_{o}$ at regions between two injection flows become higher than the centerline downstream regions of injection holes due to the strong interaction of formed horseshoe vortices between neighboring holes. This phenomenon indicates the instantaneous disturbance of mainstream caused by the injection flows ejected through film cooling holes.

At low blowing ratio, the injection flows stay close to the convex surface because of the lower momentum ratio. Therefore, the injection flows increase the surface heat transfer effect on convex surface. As the blowing ratio increases, the ejected jets will lift-off the surface immediately after leaving the injection holes, and form the lower heat transfer value than $M=0.5$ even at low $X / D$ region. At high blowing ratio, the counter-rotating vortex pairs formed within the ejected jet are strong. The interactions among the vortices of neighboring injection flows provide high $h / h_{o}$ on the regions between injection flows. Fric et al. (1994) observed that the injection flows will lift away from the wall immediately after the injection flows exit the injection holes because both injection flow mass flux and momentum ratio are increased when blowing ratio increases. The horseshoe vortex will be formed when the mainstream encounters the ejected jets, and wake vortices will appear at downstream regions along centerline of injection holes. A reproduction of Figure 1 in the work of Fric et al. is given as Figure 5. Both the horseshoe vortex and wake vortices increase the surface heat transfer. It is shown that $h / h_{o}$ at most regions of $M=2.0$ is higher than $M=1.5$ in Figure 4 of the present study.

Figure 6 illustrates the effect of blowing ratio on the spanwise averaged heat transfer ratio $\left(\overline{h / h_{o}}\right)$ for $2.9<X / D<25$. The spanwise averaged results for regions of $X / D<2.9$ are not provided in this work since the one-dimensional heat conduction model used in data analysis might not be applicable to the three-dimensional thermal-fluid behavior around these injection holes.

For $X / D>8$, it is obvious that the value of $\overline{h / h_{o}}$ rises at blowing ratios of 0.5 and 0.75 , because injection flows of lower mass and momentum ratio will be constrained against the wall by mainstream. Therefore, $\overline{h / h_{o}}$ is higher at lower blowing ratio at most measured regions as shown in Figure 6. The injection flows ejected into the boundary layer of mainstream would increase the 


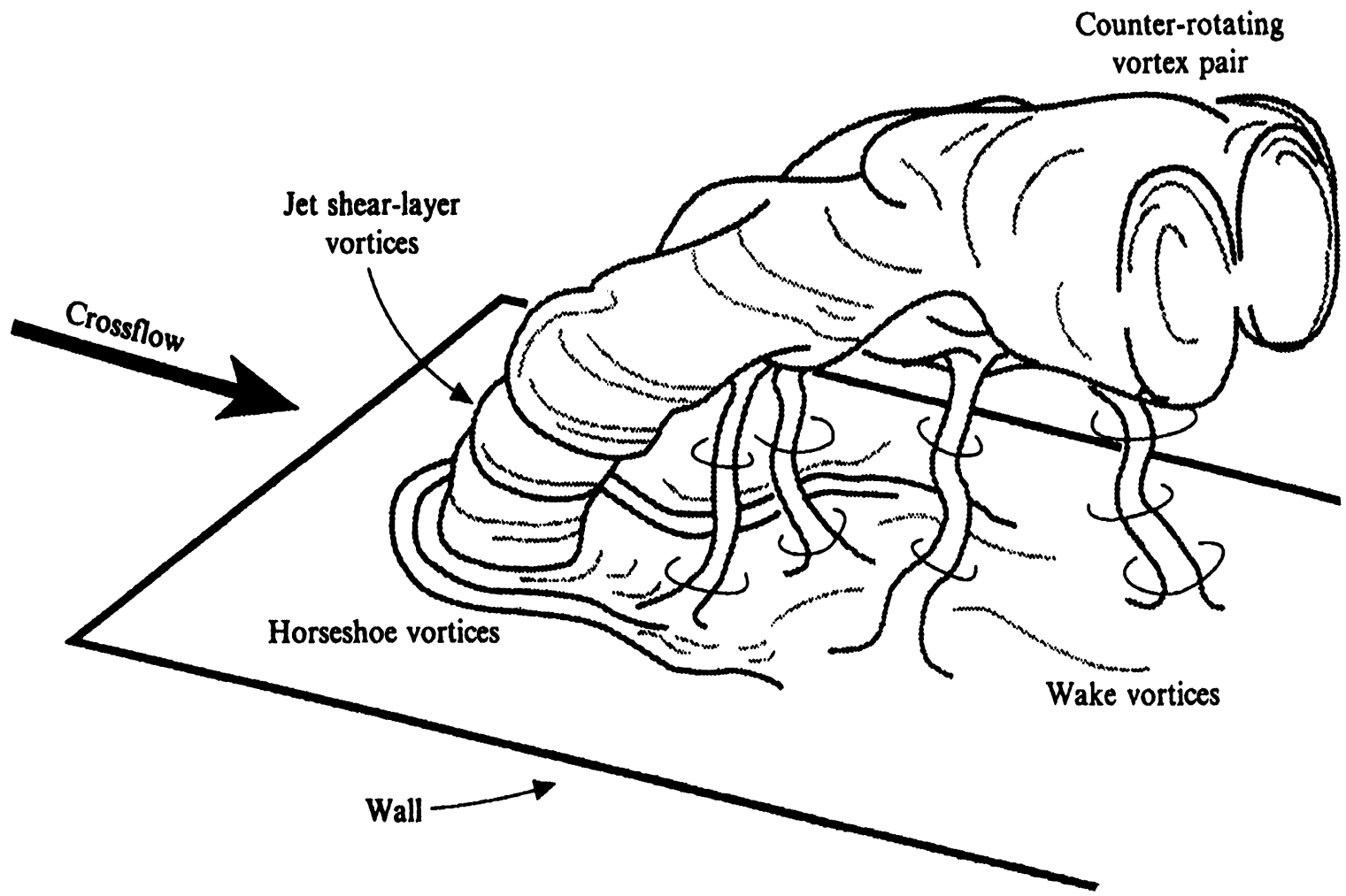

FIGURE 5 Cartoon depicting four types of vortical structure associated with the transverse-jet near field. (reproduction of Fig. 1 in Fric et al., 1994.)

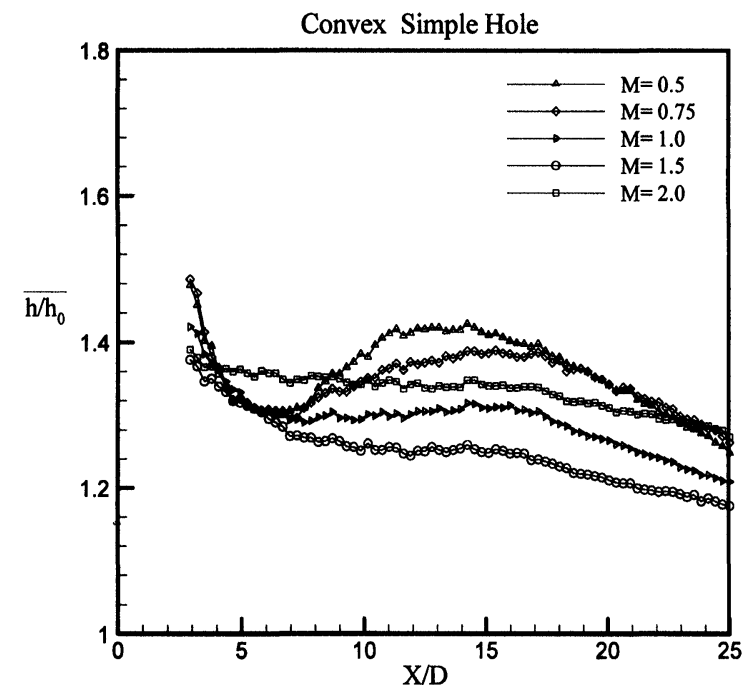

FIGURE 6 Effect of blowing ratio on the spanwise averaged heat transfer coefficient. mainstream boundary layer thickness. This effect of increasing boundary layer thickness will become more obvious as the blowing ratio increases, and thereby decreases the $\overline{h / h_{o}}$ at $X / D>8$. As blowing ratio is increased to 2.0 , the injection flows of relatively high momentum tend to lift-off the convex surface after injection. Each injection flow becomes an inclined cylinder for the mainstream. Hence, a horseshoe vortex will be formed around the injection flow and also wake vortices will be induced (Fric et al., 1994). The interactions of the horseshoe vortices among the injection flows are strong and will increase the surface heat transfer at regions between holes. Moreover, the wake vortices will increase the surface heat transfer at regions downstream of holes. Therefore, the $\overline{h / h_{o}}$ at most measured regions increase as blowing ratio is increased to 2.0 .

At low blowing ratio, Goldstein and Stone (1997) observed similar phenomena from the flow 
visualization results. In curved flows, a crossstream pressure gradient that holds low momentum fluid particles against the convex surface exists. The effect on the mainstream boundary layer is to suppress mixing and entrainment in the convex surface boundary layer. Therefore, on the convex surface, injection flow ejected at low blowing ratio stays close to the surface and remains in-line with the injection hole.

Figure 7 shows the detailed distributions of local film cooling effectiveness ( $\eta$ ) at $M=0.5$ to $M=2.0$. At blowing ratio of 0.5 , the injection flows stay close to the convex surface and block the mainstream from the surface, thereby increasing the film cooling effectiveness gradually as shown in Figure 7(a). The protection of the injection flows on the convex surface against the hot gas is decreasing with increased blowing ratio. When blowing ratio increases to 1.0 , the film cooling effectiveness of regions between holes becomes less than 0.05 for $3<X / D<10$. The regions with $\eta<0.05$ extends to $X / D \approx 14$ at blowing ratio of 2.0, as demonstrated in Figure 7(d). The momentum flux ratio demonstrates the cause of the injection flows leaving the convex surface at higher blowing ratio. The large normal component of

(a)

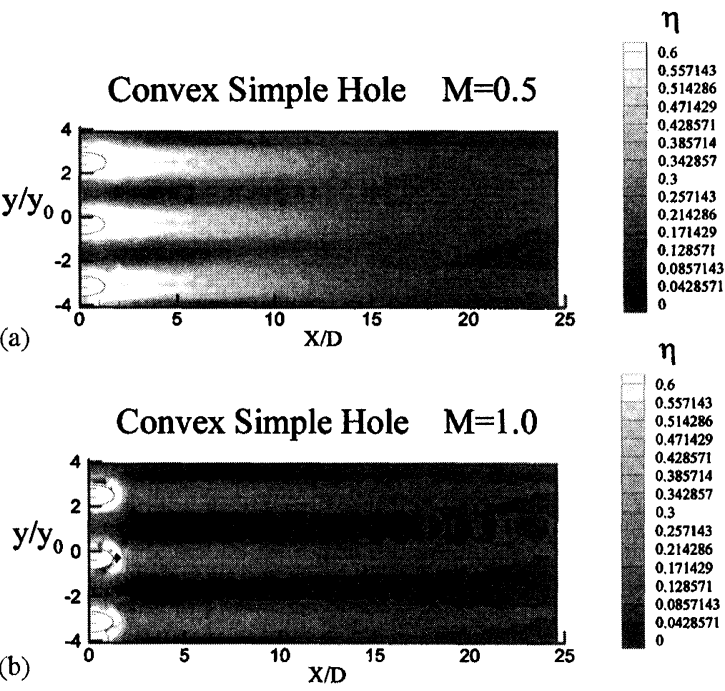

FIGURE 7 Local film cooling effectiveness distributions at (a) $M=0.5$; (b) $M=1.0$; (c) $M=1.5$; (d) $M=2.0$.

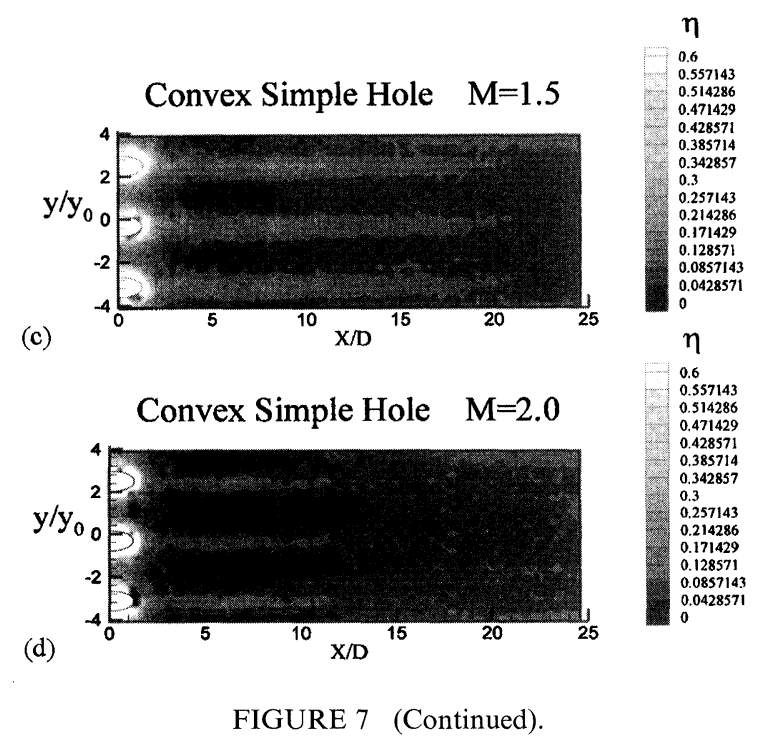

momentum of injection flows $\left(I \sin ^{2} \gamma\right)$ pulls the injection flows away from the convex surface and degrades the film cooling effectiveness on the surface. Although the increase in mass per unit area at higher blowing ratio tends to increase $\eta$, the effect of decreasing $\eta$ caused by strong vortices will be more severe.

The results of spanwise averaged film cooling effectiveness $(\bar{\eta})$ at various blowing ratios are shown in Figure 8. It is shown that $\bar{\eta}$ decreases as blowing ratio increases. At blowing ratio of 0.5, the spanwise averaged effectiveness is the best among all tested blowing ratios. At downstream regions, mixing with the mainstream dilutes the injection flows, thereby lower $\bar{\eta}$ obtained. Also, the lower injection flow mass flux of injection flow for $M=0.5$ causes the steep descent in $\bar{\eta}$ at $X / D>8$. In general, higher blowing ratios produce increased mass per unit area, larger momentum but greater jet penetration. Increase of mass can increase the spanwise averaged film cooling effectiveness at downstream regions and provide an even film coverage along $X / D$. As shown in Figure 7(d), at blowing ratio of 2.0, the increase of $\eta$ for $X / D>15$ at regions between injection flows shows that the reattachments of injection flows have occurred, so increasing the $\bar{\eta}$ far downstream in Figure 8. 


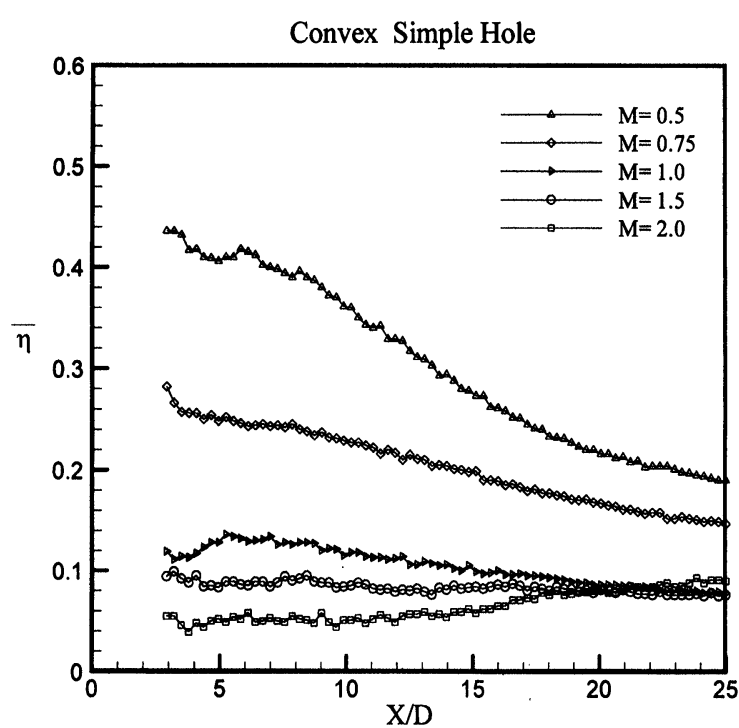

FIGURE 8 Effect of blowing ratio on the spanwise averaged film cooling effectiveness.

For the film cooling effectiveness, Schwarz et al. (1991) noted that the effect of cross-stream pressure gradient greatly overshadow the effects of the normal and tangential momentum of the jet at low blowing ratio, thereby pushing the injection flows onto the convex surface. Farther downstream, at low blowing ratios, mixing with the mainstream dilutes the coolant gas and then $\bar{\eta}$ degrades. Berhe and Patankar (1999b) mentioned that the pairs of counter-rotating vortex would be stronger at high blowing ratios. This phenomenon causes the decrease in $\bar{\eta}$ because the pairs of counter-rotating vortex lift the injection flow away from the surface and entrain the hot gases from the surroundings to the surface. For the present study, a pair of counter rotating vortices obviously decreases $\bar{\eta}$ when $M>1.0$.

The film cooling performance of test surface is best indicated by the result of spanwise averaged heat flux ratio shown in Figure 9. The $\overline{q / q_{o}}$ of values lower than 1.0 at low blowing ratio indicates better film cooling performance. At $M=0.5$ and 0.75 , the injection flows reduce the heat flux ratio effectively over most measured regions because the low normal momentum of ejected injection flows tend to lead the flows to stay

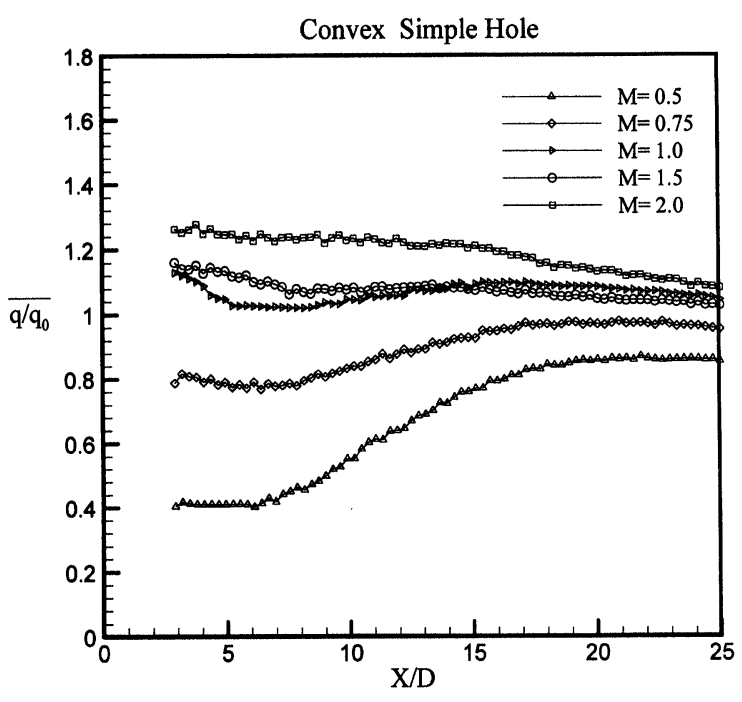

FIGURE 9 Effect of blowing ratio on the overall heat flux ratio.

close to the surface. But the low mass flux of injection flows is unable to provide film protection at further downstream, and therefore increases the magnitude of $\overline{q / q_{o}}$ at $X / D>9$. When $M$ is further increased to 1.0, 1.5 and 2.0, both the increased normal momentum and counter-rotating vortex pairs will promote lift-off and therefore perform poor protection just after injection. Furthermore, at downstream of $X / D=12$, the value of $\overline{q / q_{o}}$ at $M=1.0$ is larger than the value at $M=1.5$. This phenomenon reveals the effect of higher mass flux on surface protection at downstream regions. As a summary, at high blowing ratio, the high normal momentum of ejected injection flow promotes lift-off phenomenon and degrades the protection on the convex surface. For the tested convex surface in the present study, the low blowing ratio of 0.5 shows the best film cooling performance.

\section{CONCLUSIONS}

The present study demonstrates the influence of blowing ratio on convex surface film cooling performance. By using transient liquid crystal 
thermography, the film cooling performance of a convex surface $(2 r / D=92.5)$ with a row of straight circular holes $\left(\gamma=35^{\circ}\right)$ was investigated. Experimental results show that high blowing ratio has adverse effect on film cooling performance. At high blowing ratio, the injection flows will lift away from the surface and will also reduce the film cooling effectiveness on the convex surface. However, the larger mass of injection flow ejected into the mainstream at higher blowing ratio causes adjacent injection flows to merge far downstream of injection holes and increases the film cooling effectiveness. In the present study, injection flows are not able to offer better protection on the convex surface when $M \geq 1.0$. For the tested convex surface in the present study, the optimum blowing ratio is found to be 0.5 among the present tested blowing ratios.

\section{Acknowledgment}

The authors deeply appreciate the financial support by NSC under the grant number 86-2212-E002-080. The work in this study could not be achieved without their support.

\section{NOMENCLATURE}

$D \quad$ injection hole diameter on the inlet plane [m]

$h$ heat transfer coefficient with film injection $\left[\mathrm{W} / \mathrm{m}^{2} \mathrm{~K}\right]$

$h_{o} \quad$ baseline heat transfer coefficient without film injection $\left[\mathrm{W} / \mathrm{m}^{2} \mathrm{~K}\right]$

I momentum flux ratio $=\rho_{c} u_{c}^{2} / \rho_{m} u_{m}^{2}$

$L \quad$ length of injection hole [m]

$M \quad$ blowing ratio $=\rho_{c} u_{c} / \rho_{m} u_{m}$

$P \quad$ pitch of injection holes [m]

$q$ heat flux per unit area $\left[\mathrm{W} / \mathrm{m}^{2}\right]$

$q_{o}$ baseline heat flux per unit area without film injection $\left[\mathrm{W} / \mathrm{m}^{2}\right]$

$r$ radius of curvature of convex surface [m]

$R e_{d}$ mainstream Reynolds number based on the inlet dia-meter of injection hole $=\rho_{m} u_{m} D / \mu$ $t \quad$ time [s]

$T$ temperature $[\mathrm{K}]$

$\mathrm{Tu}$ mainstream turbulence intensity [\%]

$u$ velocity $[\mathrm{m} / \mathrm{s}]$

$X$ axial distance from the center of injection hole $[\mathrm{m}]$

$Y$ spanwise coordinate from the center of injection hole [m]

$z \quad$ coordinate axis perpendicular to the test surface [m]

\section{Greek Symbols}

$\alpha \quad$ thermal diffusivity of test surface $\left[\mathrm{m}^{2} / \mathrm{s}\right]$

$\delta_{1}$ displacement thickness [m]

$\phi \quad$ overall film cooling effectiveness $=\left(T_{w}-T_{m}\right) /$

$$
\left(T_{c}-T_{m}\right)
$$

$\gamma \quad$ injection hole angle with respect to the test surface as projected into the streamwise/ normal plane (inclination angle) [deg.]

$\eta \quad$ film cooling effectiveness

$\bar{\eta} \quad$ spanwise averaged film cooling effectiveness

$\mu$ dynamic viscosity of mainstream $[\mathrm{kg} / \mathrm{ms}]$

$\rho$ density $\left[\mathrm{kg} / \mathrm{m}^{3}\right]$

\section{Subscripts}

$c$ coolant flow ejected from the injection hole

$m$ mainstream

$s \quad$ test piece

$w \quad$ surface of test piece

$i \quad$ initial condition

\section{References}

Behle, M., Schulz, K., Leiner, W. and Fiebig, M. (1996) Colorbased Image Processing to Measure Local Temperature Distributions by Wide-Band Liquid Crystal Thermography, Applied Scientific Research, 56, 113-143.

Berhe, M. K. and Patankar, S. V. (1999a) Curvature Effects on Discrete-Hole Film Cooling, ASME Journal of Turbomachinery, 121, 781-791.

Berhe, M. K. and Patankar, S. V. (1999b) Investigation of Discrete-Hole Film Cooling Parameters using CurvedPlate Models, ASME Journal of Turbomachinery, 121, $792-803$.

Camci, C., Kim, K. and Hippensteele, S. A. (1992) A New Hue Capturing Technique for the Quantitative Interpretation of 
Liquid Crystal Images used in Convective Heat Transfer Studies, ASME J. of Turbomachinery, 114, 765-775.

Chen, P. H., Ai, D. and Lee, S. H. (1998) Effects of Compound Angle Injection on Flat-Plate Film Cooling Through a Row of Conical Holes, ASME Paper No. 98-GT-459.

Ekkad, S. V., Zapata, D. and Han, J. C. (1997a) Heat Transfer Coefficients over a Flat Surface with Air and $\mathrm{CO}_{2}$ Injection through Compound Angle Holes using a Transient Liquid Crystal Image Method, ASME J. Turbomachinery, 119, $580-586$

Ekkad, S. V., Zapata, D. and Han, J. C. (1997b) Film Effectiveness over a Flat Surface with Air and $\mathrm{CO}_{2}$ Injection through Compound Angle Holes using a Transient Liquid Crystal Image Method, ASME J. Turbomachinery, 119, 587-593.

Fric, T. F. and Roshko, A. (1994) Vortical Structure in the wake of a Transverse Jet, J. of Fluid Mech., 279, 1-47.

Goldstein, R. J. and Stone, L. D. (1997) Row-of-Holes Film Cooling of Curved Walls at Low Injection Angles, ASME J. of Turbomachinery, 119, 574-579.
Ito, S., Goldstein, R. J. and Eckert, E. R. G. (1978) Film Cooling of a Gas Turbine Blade, ASME J. of Engineering for Power, 100, 476-481.

Ko, S.-Y., Yao, Y.-Q., Xis, B. and Tsou, F.-K. (1986) Discrete-Hole Film Cooling Characteristics over Concave and Convex Surfaces, Heat Transfer 1986, Proceeding of 8th International Heat Transfer Conference, San Francisco, 3, Hemisphere Publishing Corp., New York, 1297-301.

Lin, Y.-L. and Shih, T. I.-P. (1998) Computations of DiscreteHole Film Cooling over Flat and Convex Surfaces, $A S M E$ Paper, No. 98-GT-436.

Moffat, R. J. (1988) Describing the Uncertainties in Experimental Results, Experimental Thermal and Fluid Science, 1, 3-17.

Schwarz, S. G., Goldstein, R. J. and Eckert, E. R. G. (1991) The Influence of Curvature on Film Cooling Performance, ASME J. of Turbomachinery, 113, 472-478.

Vedula, R. J. and Metzger, D. E. (1991) A Method for the Simultaneous Determination of Local Effectiveness and Heat Transfer Distributions in Three-Temperature Convection Situations, ASME Paper, No. 91-GT-345. 

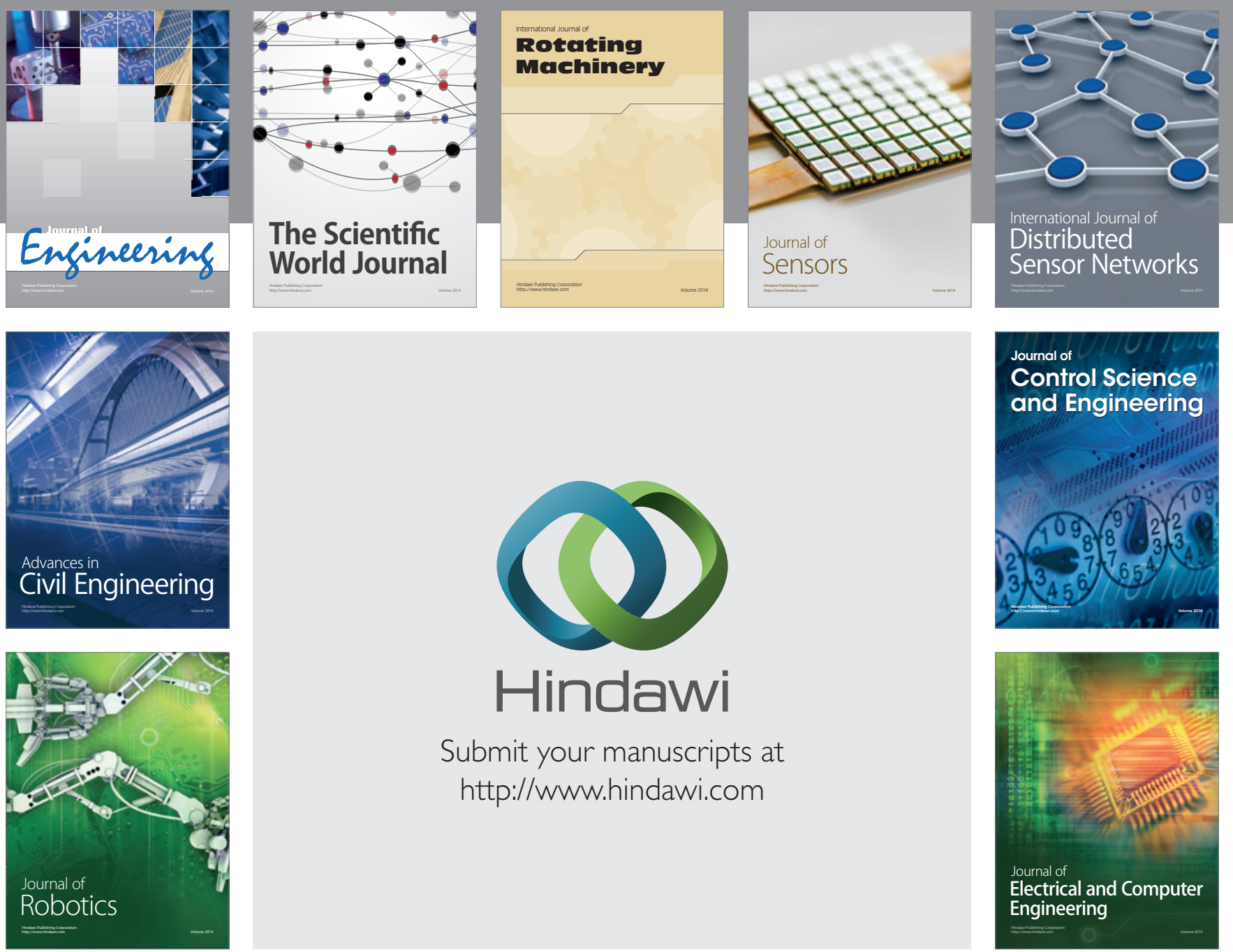

Submit your manuscripts at

http://www.hindawi.com
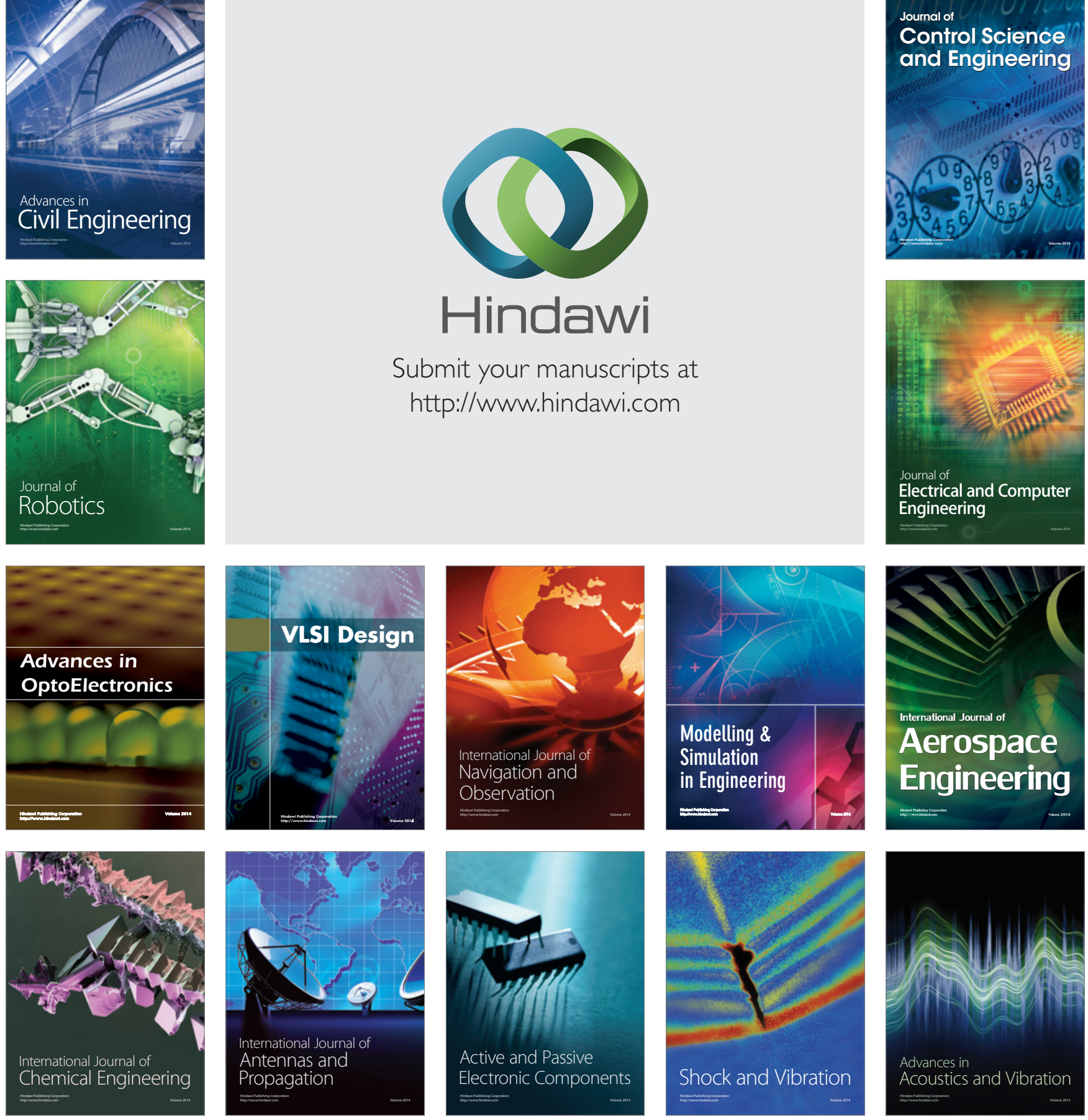